\title{
Torpedo maculopathy: disease spectrum and associated choroidal neovascularisation in a paediatric population
}

\author{
Katherine Shirley ${ }^{1} \cdot$ Marie O'Neill $^{1} \cdot$ Rachel Gamble $^{1} \cdot$ Anne Ramsey $^{2} \cdot$ Eibhlin McLoone $^{1}$ \\ Received: 29 August 2017 / Revised: 16 January 2018 / Accepted: 19 January 2018 / Published online: 20 March 2018 \\ (c) The Royal College of Ophthalmologists 2018
}

\begin{abstract}
Purpose Torpedo maculopathy is a rare lesion of the retinal pigment epithelium. This study set out to look at these lesions in the paediatric population and determine the spectrum and features of the disease.

Methods The paediatric ophthalmology database was used to identify eight children with torpedo maculopathy between 2012 and 2017. Fundal images and optical coherence tomography (OCT) was used to analyse the cases.

Results Eight patients with torpedo maculopathy were identified, making the prevalence approximately 2 per 100,000 population under 16 years old. The OCT images were classified using the previously described subtypes: Type 1 in patients 5 and 6 and Type 2 in patients 1,2,3,4 and 8. The average age of presentation of Type 1 and Type 2 lesions was 8 and 7 years old respectively. We also report patient 8 , who is the youngest reported case of choroidal neovascular membrane associated with torpedo maculopathy. Good anatomical response to a single injection of anti-Vegf was shown.

Conclusions This study is the first case series of torpedo maculopathy in the paediatric population. Contrary to previous reports of two distinct types of lesion on OCT representing different stages of the same disease, our case series indicates that Type 1 and Type 2 lesions are in fact different phenotypic entities both of which can occur at a young age. We also present the associated risk of choroidal neovascular membrane formation which is an important consideration for long term follow-up.
\end{abstract}

\section{Introduction}

Torpedo maculopathy (TM) is a rare, benign lesion of the retinal pigment epithelium (RPE). It was described in 1992 by Roseman and Gass, as a hypo-pigmented neavus of the RPE [1]. It is commonly diagnosed incidentally as patients are usually asymptomatic. Characteristically, it is a unilateral hypo-pigmented lesion temporal to the fovea with a tip pointing towards the central macula [2]. It is believed that this lesion is congenital and it has been hypothesised that it relates to the foetal temporal macular "bulge" that normally occurs at the same site as the lesions at around 4 months to 6 months gestation [3]. Generally, TM is felt to

Katherine Shirley

katherine.shirley@belfasttrust.hscni.net

1 Department of Ophthalmology, Royal Victoria Hospital, Belfast, Northern Ireland, UK

2 Ophthalmic imaging, Department of Ophthalmology, Royal Victoria Hospital, Belfast, Northern Ireland, UK be a non-progressive lesion, although occasionally the size of the lesion has shown documented increase [4]. To the authors knowledge, there have only been two reports of choroidal neovascularization (CNV) associated with TM, described in 2011 by Gass and in 2017 by Jurjevic [5, 6]. The aim of this case series is to demonstrate the spectrum of clinical appearance, the patient demographics, coinciding systemic conditions and variable optical coherence tomography (OCT) appearance in a paediatric population. In addition, we report the youngest case of TM associated $\mathrm{CNV}$ which was treated with anti- vascular endothelial growth factor (Vegf).

\section{Methods}

Analysis of the Belfast Health and Social Care Trust (BHSCT) secure paediatric ophthalmology database identified a total of eight children aged less than 16 years diagnosed with TM between 2012 and 2017. The medical records for these patients were reviewed and patient demographics, clinical presentation and medical history 
Table 1 Patient demographics and clinical presentation

\begin{tabular}{|c|c|c|c|c|c|c|c|c|}
\hline $\begin{array}{l}\text { Patient } \\
\text { OCT findings type }\end{array}$ & $\begin{array}{l}1 \\
\text { Type } 2\end{array}$ & $\begin{array}{l}2 \\
\text { Type } 2\end{array}$ & $\begin{array}{l}3 \\
\text { Type } 2\end{array}$ & $\begin{array}{l}4 \\
\text { Type } 2\end{array}$ & $\begin{array}{l}5 \\
\text { Type } 1\end{array}$ & $\begin{array}{l}6 \\
\text { Type } 1\end{array}$ & $\begin{array}{l}7 \\
\text { No OCT }\end{array}$ & $\begin{array}{l}8 \\
\text { Type } 2\end{array}$ \\
\hline Gender & Male & Female & Female & Female & Male & Male & Female & Female \\
\hline Age at presentation & 5 y.o & 5 y.o & 12 y.o & 5 y.o & 11 у.о & 5 y.o & 3 у.о & 15 y.o \\
\hline Eye involved & OS & OS & OS & OD & OD & OS & OS & OD \\
\hline $\begin{array}{l}\text { Visual acuity affected } \\
\text { eye }\end{array}$ & $6 / 9$ & $6 / 9$ & $6 / 5$ & $6 / 6$ & $6 / 6$ & $6 / 9.5$ & $6 / 12$ & $6 / 6$ \\
\hline Symptoms & Nil & Nil & Nil & Nil & Nil & Nil & Nil & $\begin{array}{l}\text { Inferior visual field } \\
\text { defect }\end{array}$ \\
\hline Past medical health & Vitiligo & $\begin{array}{l}\text { Bilateral } \\
\text { Hypermetropia }\end{array}$ & $\begin{array}{l}\text { Asthma } \\
\text { IBS }\end{array}$ & Nil & Nil & $\begin{array}{l}\text { Right } \\
\text { Amblyopia } \\
\text { Asthma } \\
\text { Autism } \\
\text { IDA }\end{array}$ & $\begin{array}{l}\text { Divergent } \\
\text { squint }\end{array}$ & Nil \\
\hline
\end{tabular}

$O D$ right eye, $O S$ left eye, y.o years old, $I B S$ irritable bowel syndrome, $I D A$ iron deficiency anaemia

were recorded. All patients underwent fundal imaging (Topcon TRC-50DX) and all, but one, had OCT performed (Optovue); the youngest child diagnosed at the age of 3 years was diagnosed in 2013 before handheld OCT was available in the department. Full approval was obtained from the Belfast Health and Social Care Trust Standards, Quality and Audit department. Information was managed in accordance with the BHSCT guidance on data protection.

\section{Results}

Patient demographics are summarised in Table 1. In our series there was a slight female preponderance. The mean age at diagnosis was 8 years old (range: 3-15). All cases were unilateral; with three presenting with lesions in the right eye and five with lesions in the left eye. All but one patient were asymptomatic on presentation. The symptomatic patient had an inferior visual field defect due to associated CNV. Visual acuity in the affected eye ranged from $6 / 5$ to $6 / 12$ Snellen. Two patients had other ocular conditions leading to reduced visual acuity. One had bilateral hypermetropia, the other had a divergent squint.

All lesions were hypo-pigmented to varying degrees and located in the temporal macula (Fig. 1). All lesions had an oval shape, being elongated in the horizontal axis. There were variable degrees of associated hyper-pigmented RPE change surrounding the lesions. Patients 1, 4 and 6 had little or no hyperpigmentation and quite well-defined margins of the TM lesion. Patient 5 had a well-defined TM lesion with hypo-pigmented centre and fine hyperpigmentation delineating the edge. $\mathrm{He}$ also had a smaller separate lesion about one disc diameter temporally from the TM lesion.
This lesion was round and flat with hyperpigmentation both centrally and delineating the main area of hypopigmentation. Patients 2, 3 and 7 had less uniform pigmentation with an irregular appearance and characteristically a denser hyper-pigmented 'tail' temporally. The last patient, patient 8 , had evidence of some hyperpigmentation delineating the lesion but also the green hue of a CNV membrane at the nasal edge of the TM lesion with associated intra-retinal fluid (Fig. 2a).

OCT performed on seven patients again showed variation in appearance (Fig. 1). Patients 1, 3 and 6 had normal inner retinal structures but loss of the ellipsoid zone, and thinning of the outer nuclear layer, to varying degrees. Patient 2 had a similar appearance on OCT but also demonstrated choroidal excavation to a larger degree. Patient 5 had normal inner retina but localised broadening and attenuation of the interdigitation zone resulting in elevation of the external limiting membrane and ellipsoid zone. Patient 4 showed a cavitation defect of the outer retina with thinning of the outer nuclear layer and loss of both the ellipsoid and interdigitation zone resulting in a sub-retinal cleft. At time of initial presentation, OCT findings of the main TM lesion in patient 8 are similar to patients 1,3 and 6 with normal inner retinal structures but loss of the ellipsoid zone, and thinning of the outer nuclear layer (Fig. 2d). Nasal to this there is cystic intra-retinal fluid and a small area of sub-retinal fluid due to associated CNV. Fundus fluorescein angiography (FFA) on patient 8 demonstrated diffuse late leakage from the membrane adjacent to the window defect created by the TM (Fig. 2b). A follow-up OCT 2 months post a single anti-Vegf intravitreal injection of Ranibizumab (Fig. 2e) showed good response to treatment with resolution of the sub-retinal and intra-retinal fluid. Visual acuity had improved to $6 / 5$ at this time and the inferior scotoma had resolved. 
Fig. 1 Fundal photographs and OCT imaging for patients $1-7$

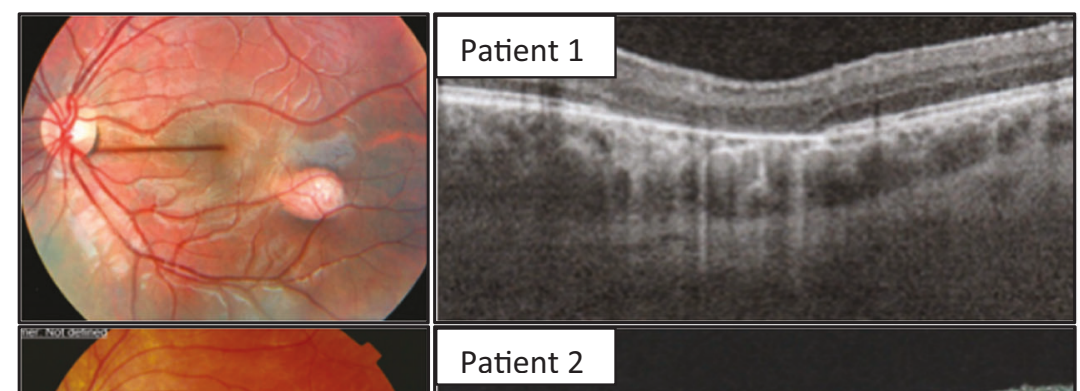

Patient 2
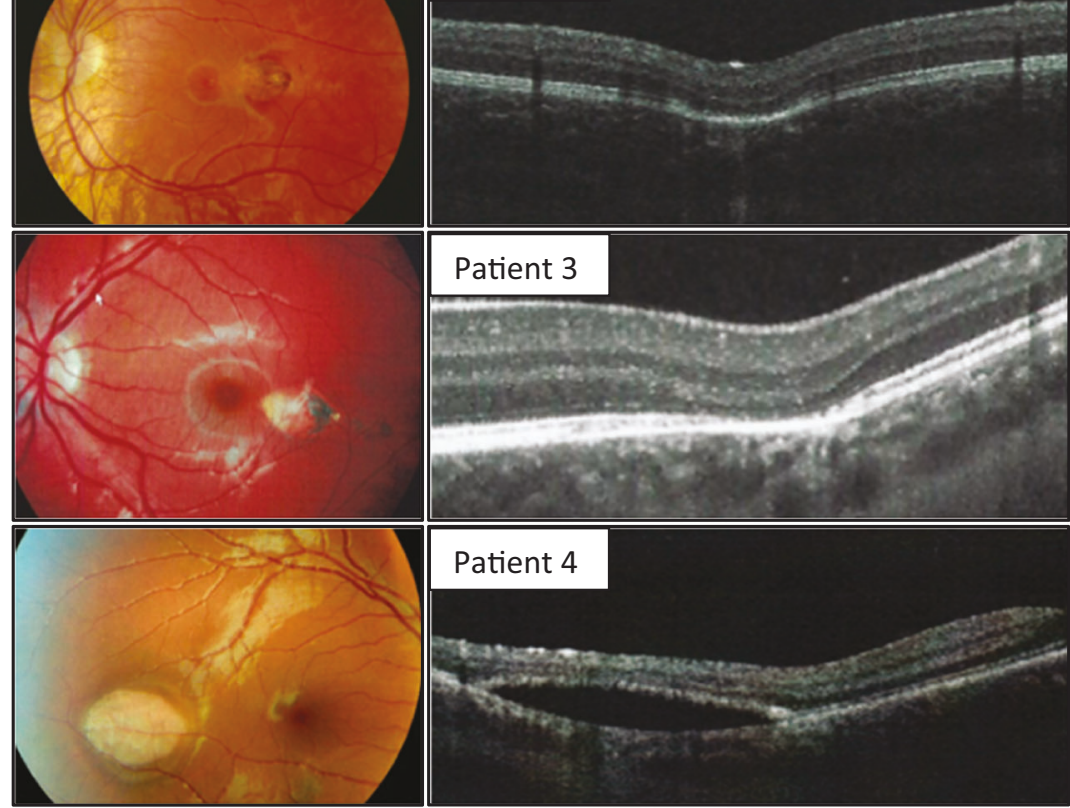

Patient 4
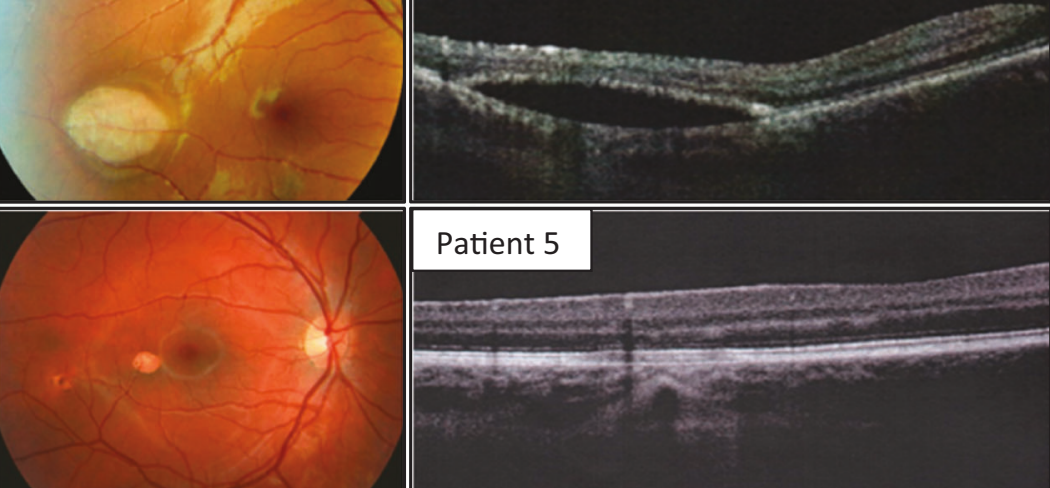

\section{Patient 5}

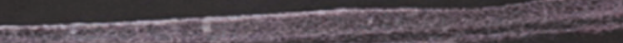

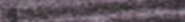

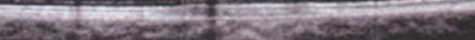

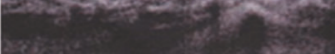

Patient 6
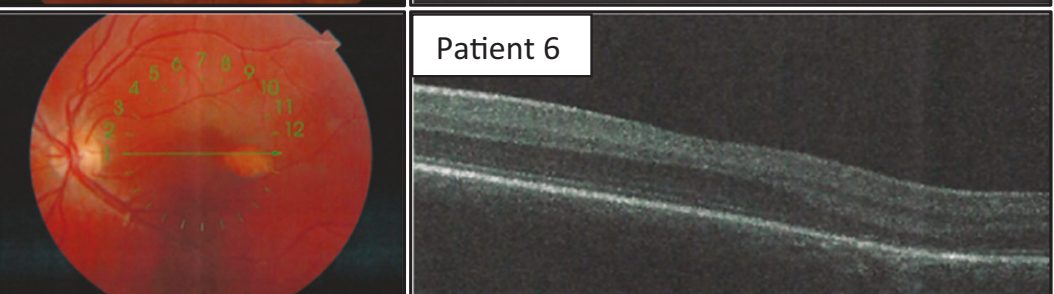

Patient 7

No OCT as aged 3yo and unable to cooperate with testing. 


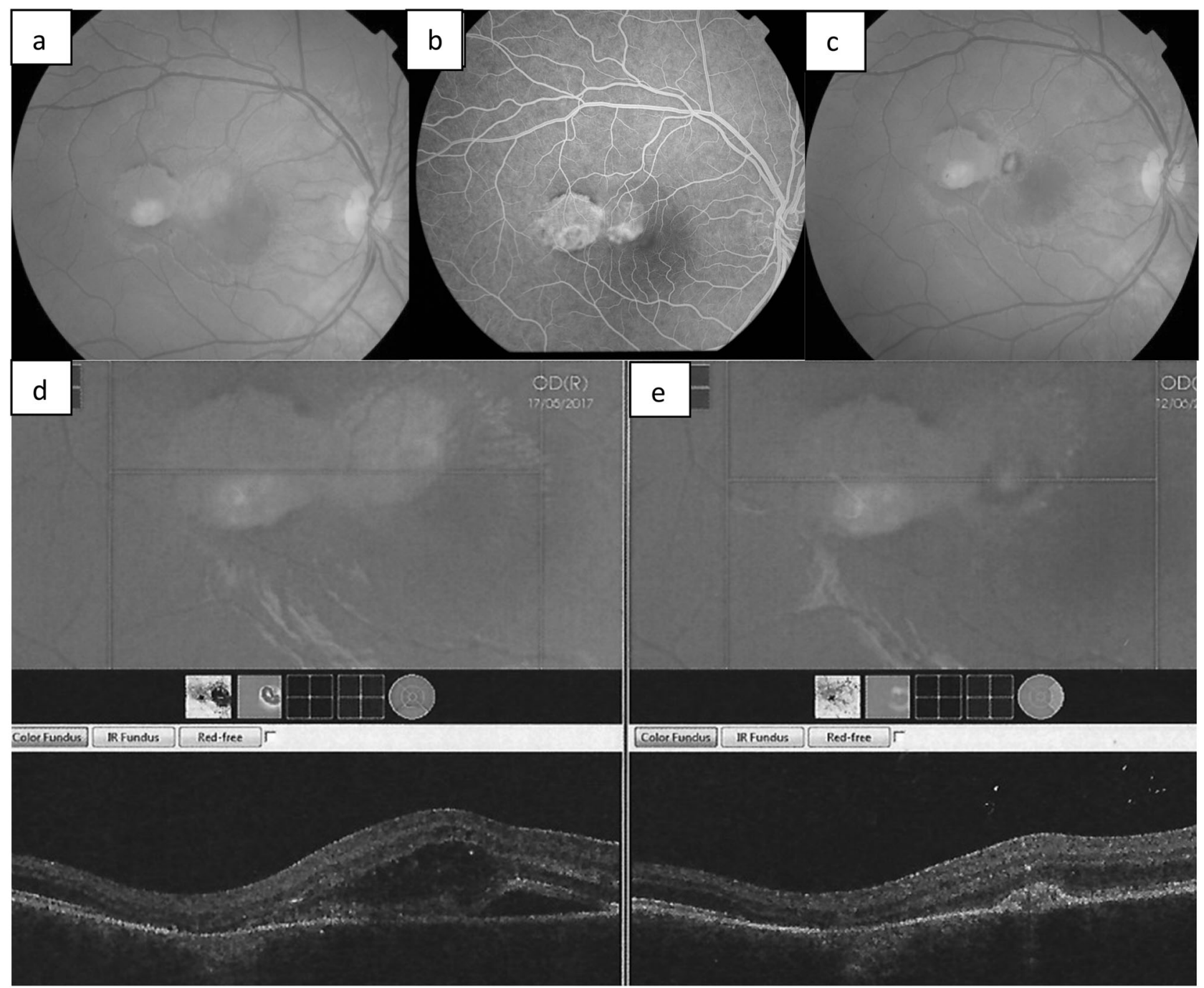

Fig. 2 Fundal imaging of patient 8. a Colour fundal photography right eye showing a torpedo maculopathy with a nasal choroidal neovascular membrane (CNVM). b Fundus fluorescein angiography showing CNVM leakage. c Colour fundal photograph 2 months post anti-VEGF

\section{Discussion}

TM is classically an incidental finding in asymptomatic patients. As a result, its detection is rare and prevalence data is unknown. Our patient cohort is population-based. According to migration statistics, immigration in Northern Ireland (NI) is greater than emigration making our population ideal for epidemiological studies. The catchment area for the regional paediatric ophthalmology centre in Belfast encompasses most of Northern Ireland (NI) for which the population of under 16 year olds in 2016 was 388,001 [7]. Therefore, the prevalence of TM in NI is approximately 2 per 100,000 population under 16 year olds. However, as TM is largely an asymptomatic condition we regognise that this is likely an underestimate of the prevalence. d OCT of torpedo maculopathy lesion and CNVM at presentation e OCT of torpedo maculopathy lesion and CNVM 2 months post antiVegf injection. A full colour version of this figure is available at the Eye journal online

The aetiology of TM is unknown, although many hypotheses have been suggested. Pian et al. [8] suggested the lesion could be a developmental defect in the nerve fibre layer at the horizontal raphe. Teitelbaum et al. [9] suggested it may represent a disturbance to the choroidal development and vasculature. Shields et al. [3] postulated a possible defect in RPE development in the foetal temporal bulge as a cause for TM.

Although histopathological studies have not been performed on these lesions, new technology including OCT has led to a better understanding of their morphology. Wong et al classified TM lesions into two distinct types based on OCT appearance [10]; Type 1 lesions which show a pattern of attenuation of outer retinal structures without outer retinal cavitation (as demonstrated by patients 5 and 6 in our cohort) and Type 2 lesions which show a pattern of 
Fig. 3 OCT imaging of satellite lesion for patient 5

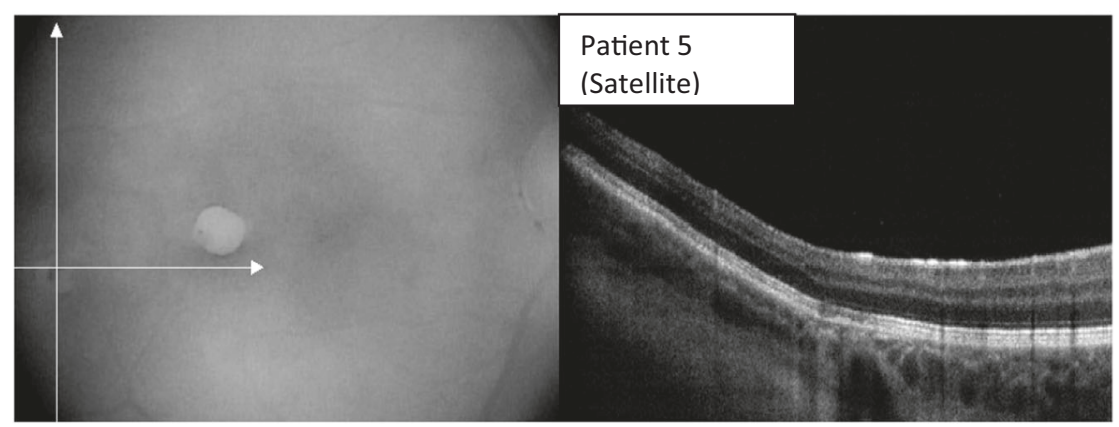

attenuation of outer retinal structures and outer retinal cavitation with neurosensory elevation (as demonstrated by patients 1, 2, 3, 4 and 8 in our case series). Wong et al. [10] hypothesised that these two destinct OCT subtypes represent different stages of TM that evolve from Type 1 to Type 2 over several decades. They reported Type 2 lesions in older patients with an average age at presentation of 39 years compared to 17 years for Type 1 lesions [10]. Contrary to Wong et al's findings, we present three patients in our series who all presented aged 5 years old with type 2 lesions on OCT (patients 1, 2 and 4). To the authors' knowledge, these are the youngest patients described with a type 2 lesion on OCT. In our case series of children under 16 years old with TM the average age of presentation of Type 1 and Type 2 lesions was 8 and 7 years old respectively. Suggesting that these represent different phenotypic presentations rather than different stages of the same disease. With the evolution of hand held OCT allowing younger children to undergo assessment, the differentiation of TM lesions may become clearer. This has clinical importance as it has been previously reported that excavation of the fundus in this form of TM lesion can result in a corresponding scotoma [11]. Recognition of this may be important for those lesions in close proximity to the fovea.

Although TM is classically described as a solitary, oval lesion, variably pigmented and located in the macula [1], satellite lesions can also occur. In particular we highlight patient 5 in our cohort who demonstrates a small satellite lesion temporal to the main TM lesion (Fig. 1). Reviewing previously documented cases in the literature we can identify 3 other clinical cases that have similar presentations [6, 10]. All satellite lesions to date are located temporally and are small, round and flat as seen in patient 5. OCT imaging of these lesions has previously been described as normal however OCT of the satellite lesion in our patient reveals similar changes to the main TM lesion with normal inner retina but localised broadening and attenuation of the interdigitation zone resulting in elevation of the external limiting membrane and ellipsoid zone [6] (Fig. 3). Satellite lesions may add to our understanding of the morphology of these lesions as they appear to be an extension of the main lesion. This feature along with the variable phenotypes in young patients would better support a congenital defect of the RPE during foetal development that is non-progressive. When Streeten studied the foetal RPE development he documented that in early development there was a coneshaped bulge in the temporal macula where TM lesions are found [12]. This staphylomatous lesion gradually lessens between 6 months and 8.5 months. We hypothesis that prenatal insults to development at different points over these last 3 months may lead to these different clinical phenotypes.

As previously mentioned, TM is felt to be an asymptomatic, benign condition and many patients are discharged from routine follow-up. However, a recent case of a 34year-old patient with CNV related to TM was published by a group in Switzerland [6]. It was only described once prior to this in Gass' Atlas of macular diseases [5]. To the authors' knowledge, patient 8 is only the third reported case and is the youngest case of TM complicated by CNV. Our patient initially presented at age 15 with inferior field distortion and was found to have an active CNV membrane on fluorescein angiography. Our patient has shown a good anatomical response to a single injection of Ranibizumab, anti-Vegf but long term prognosis is uncertain. OCT has demonstrated that the outer retina and RPE are affected by TM lesions and therefore we suggest that patients with TM lesions are susceptible to the development of CNV and need to be advised accordingly.

\section{Conclusion}

We present the first case series of TM in a paediatric population. Contrary to previous reports of two distinct types of TM on OCT representing different stages of the same disease, our case series indicates that Type 1 and Type 2 TM lesions are in fact different phenotypic entities both of which can occur at a young age. Although TM is in general a benign, asymptomatic finding there is an associated risk of choroidal neovascular membrane formation as demonstrated by one of our young patients. It is important that physicians and patients are aware of this potential risk and as younger patients are unlikely to report symptoms of CNV they may require long term follow-up. 


\section{Summary}

\section{What was known before}

- Torpedo Maculopathy is a rare, benign lesion of the retinal pigment epithelium.

- Only one published case of associated choroidal neovascular membrane.

- Hypothesized two different OCT imaging types that were an evolution of the disease over time.

\section{What this study adds}

- First Paediatric case series of Torpedo Maculopathy, estimating a prevalence of 2 per 100,000.

- This study shows that both types of OCT appearance are seen in young patients indicating 2 different phenotypic presentations of the disease rather than evolution over time.

- First paediatric case of Choroidal neovascular membrane associated with Torpedo Maculopathy responding well to anti-VEFG.

\section{Compliance with ethical standards}

Conflict of interest The authors declare that they have no conflict of interest.

\section{References}

1. Roseman RL, Gass JDM. Solitary hypo-pigmented nevus of the retinal pigment epithelium in the macula. Arch Ophthalmol. 1992;110:1358-9.

2. Golchet P, Jampol L, et al. Torpedo maculopathy. Br J Ophthalmol. 2010;94:302-6.

3. Shields C, Guzman J, Shapiro M, et al. Torpedo maculopathy at the site of the fetal "bulge". Arch Ophthalmol. 2010;128:499-501.

4. Sanabria MR, Coco RM, Sanchidrian M. OCT findings in torpedo maculopathy. Retin Cases Brief Rep. 2008;2:109-11.

5. Agarwal A. Gass' Atlas of macular diseases. 1076. Toronto, Canada: Elsevier Health Sciences; 2011.

6. Jurevic D, Boni C, Barthelmes D, et al. Torpedo maculopathy associated with choroidal neovascularisation. Klin Mon Augenheilkd. 2017;234:508-14.

7. Northern Ireland Statistics and Research Agency. 2016 Mid year population estimates for Northern Ireland. 2017. www.nisra.gov.uk.

8. Pian D, Ferrucci S, Anderson SF, Wu C. Paramacular coloboma. Optom Vis Sci. 2003;80:556-63.

9. Teitelbaum BA, Hachey DL, Messner LV. Torpedo maculopathy. J Am Optom Assoc. 1997;68:373-6.

10. Wong E, Fraser-Bell S, Hunyor A, Chen F. Novel optical coherence tomography classification of torpedo maculopathy. Clin Exp Ophthalmol. 2015;43:342-8.

11. Trevino R, Kiani S, Raveendranathan R. The expanding clinical spectrum of torpedo maculopathy. Optom Vision Sci. 2014;91: S71-S78.

12. Streeten BW. Development of the human retinal pigment epithelium and the posterior segment. Arch Ophthalmol. 1969;81: 383-94. 